\title{
Altered Functional Connectivity in Resting State Networks in Tourette's Disorder
}

\begin{abstract}
Siyan Fan ${ }^{1,2,3 *}$, Odile A. van den Heuvel2,3,4, Danielle C. Cath ${ }^{1,5}$, Stella J. de Wit ${ }^{3}$, Chris Vriend ${ }^{2,3}$, Dick J. Veltman ${ }^{3}$ and Ysbrand D. van der Werf ${ }^{2}$

${ }^{1}$ Division of Social and Behavioural Science, Utrecht University, Utrecht, Netherlands, ${ }^{2}$ Department of Anatomy \& Neurosciences, Amsterdam Neuroscience, Amsterdam UMC, Vrije Universiteit Amsterdam, Amsterdam, Netherlands, ${ }^{3}$ Department of Psychiatry, Amsterdam Neuroscience, Amsterdam UMC, Vrije Universiteit Amsterdam, Amsterdam, Netherlands, ${ }^{4}$ The OCD Team, Haukeland University Hospital, Bergen, Norway, ${ }^{5}$ Department of Psychiatry and Rob Giel Research Center (RGOC), University Medical Center Groningen, University of Groningen, Groningen, Netherlands
\end{abstract}

Introduction: Brain regions are anatomically and functionally interconnected in order to facilitate important functions like cognition and movement. It remains incompletely understood how brain connectivity contributes to the pathophysiology of Tourette's disorder (TD). By using resting-state functional MRI, we aimed to identify alterations in the default mode network (DMN), frontal-parietal network (FPN), sensori-motor network $(\mathrm{SMN})$, and salience network (SN) in TD compared with healthy control $(\mathrm{HC})$ subjects.

Method: In 23 adult TD patients and $22 \mathrm{HC}$, 3T-MRI resting-state scans were obtained. Independent component analysis was performed comparing TD and $\mathrm{HC}$ to investigate connectivity patterns within and between resting-state networks.

OPEN ACCESS

Edited by:

Juliana Yordanova, Institute of Neurobiology (BAS),

Bulgaria

Reviewed by: Wei Liao,

University of Electronic Science and Technology of China, China Arun Bokde,

Trinity College, Dublin, Ireland

${ }^{*}$ Correspondence: Siyan Fan s.fan@vumc.nl, siyan.fan@yale.edu

Received: 14 January 2018

Accepted: 23 August 2018

Published: 18 September 2018

Citation:

Fan S, van den Heuvel OA, Cath DC, de Wit SJ, Vriend C, Veltman DJ and van der Werf YD (2018) Altered

Functional Connectivity in Resting

State Networks in Tourette's Disorder.

Front. Hum. Neurosci. 12:363.

doi: 10.3389/fnhum.2018.00363
Results: TD patients showed higher involvement of the dorsal medial prefrontal cortex in the connectivity of the DMN and less involvement of the inferior parietal cortex in the connectivity of the FPN when compared to HC. Moreover, TD patients showed a stronger coupling between DMN and left FPN than HC. Finally, in TD patients, functional connectivity within DMN correlated negatively with tic severity.

Conclusion: We tentatively interpret the increased functional connectivity within DMN in TD patients as compensatory to the lower functional connectivity within left FPN. The stronger coupling between DMN and left FPN, together with the finding that higher DMN intrinsic connectivity is associated with lower tic severity would indicate that DMN is recruited to exert motor inhibition.

Keywords: resting-Ss, Tourette Syndrome, functional connectivity (FC), default mode network (DMN), frontalparietal network

\section{INTRODUCTION}

Tourette's disorder (TD) is a neuropsychiatric disorder characterized by involuntary, repetitive and compulsive movements, expressed as motor, phonic, and complex tics (Freeman, 2013). The neurobiological model of TD suggests a key role for the frontal cortico-striatal-thalamo-cortical (CSTC) circuits (Leckman, 2002; Milad and Rauch, 2012; Ji et al., 2016). These CSTC circuits originate from specific regions of the frontal cortex and project to the basal ganglia via direct or indirect pathways, culminating in the thalamus before projecting back to the frontal regions 
(Alexander et al., 1986). In recent years, this model has been extended to include interactions at the cortical level, specifically involving the fronto-parietal network (FPN), based on emerging functional and structural neuroimaging evidence for its relevance to the pathophysiology of TD (Ganos et al., 2013; Worbe et al., 2015). Whereas the brain can be considered a sophisticated multinetwork organ, anatomically and functionally interconnected in order to optimally accomplish, e.g., cognitive and motor functions (van den Heuvel and Hulshoff Pol, 2010), it is unclear whether CSTC circuit abnormalities in TD interfere with known resting-state networks.

Resting-state functional magnetic resonance imaging (rs$\mathrm{fMRI}$ ) is a popular neuroimaging method for measuring spontaneous slow blood-oxygen level-dependent (BOLD) fluctuations that show co-activation between brain regions during resting state (Biswal et al., 1997). Several resting-state networks have been identified that display high-level functional connectivity between widespread cortical and subcortical areas. Four of these networks are specifically relevant for TD.

The default mode network (DMN) consists of the posterior cingulate cortex (PCC)/precuneus, medial frontal and inferior parietal and temporal regions (Buckner and Vincent, 2007), and is thought to be at the core of integrating cognitive and emotional processes (Greicius et al., 2003), mind-wandering (Mason et al., 2007) and monitoring the world around us (Gusnard et al., 2001); abnormal functional connectivity in the DMN has been reported in movement disorders like Parkinson's disease (Tessitore et al., 2012; Esposito et al., 2013) and Huntington's disease (Wolf et al., 2012; Werner et al., 2014). Recently, Wen et al. (2018) studied the topological organization of functional connectivity networks in TD children by using graph theory measures. Compared to $\mathrm{HC}$, pediatric TD patients showed altered topology of the DMN. Another study reported an intact DMN when adult TD patients were instructed to lie in the scanner and were allowed to tic (as comparing to no tic activity), although no direct comparison with HC was made (Neuner et al., 2014). Altered functional integrity of DMN has also been demonstrated in other neuropsychiatric disorders such as OCD (Stern et al., 2012) and ADHD (De La Fuente et al., 2013).

The fronto-parietal network, consists of the anterior prefrontal cortex, anterior cingulate cortex (ACC), anterior insula, anterior inferior parietal lobule and the caudate nucleus and likely underlies attention processing and higher order cognitive functions (Damoiseaux et al., 2006). The salience network (SN), including bilateral anterior insula, ACC, preSMA, amygdala, ventral striatum and substantia nigra, is also involved in attentional control, as well as responding to behaviorally salient events (e.g., error detection) and important for goal-directed behavior (Seeley et al., 2007; Ham et al., 2013). Church et al. (2009) investigated adolescent TD patients and used a region of interest (ROI) approach to examine functional connectivity in the FPN and the SN (cingulo-opercular network). In TD patients showed anomalous functional connections in both the FPN and the SN when compared to healthy controls (HCs). In adult TD patients, Worbe et al. (2012) quantified functional connectivity in CSTC circuits using global integration and graph theory measures. They showed overall more interactions among these anatomical regions and a global functional disorganization of all these networks in patients compared to HC (Worbe et al., 2012).

The sensorimotor network (SMN), connecting the precentral with postcentral gyri and the supplementary motor area (SMA) is involved in motor control (Damoiseaux et al., 2006). Although using different data analytical approaches, several recent studies have reported abnormalities in the SMN in TD patients. For instance, Ji et al. (2016) studied amplitude of low-frequency fluctuation (ALFF) and functional connectivity and showed abnormalities in the SMN regions in TD children compared to HC. A different study also reported on TD children, and showed altered intrinsic connectivity in the sensorimotor areas (Liao et al., 2017). Additionally, topological disruptions were also observed in the SMN in TD patients (Wen et al., 2018).

The main objective of the present study is to replicate and extend previous literature on brain functional connectivity in relation to the pathophysiology of TD in the four abovementioned specific resting-state networks (DMN, FPN, SN, and $\mathrm{SMN}$ ) by using a data-driven analytical approach - independent component analysis (ICA). We first aimed to identify alterations in functional connectivity within these networks in TD compared to HC. Second, we examined relationships between functional connectivity and disease severity in TD.

We hypothesized that altered functional connectivity would be observed in TD in the four targeted networks based on the previous literature; as these previous studies adopted different methodological approaches to quantify functional connectivity measures, we did not have a prediction on the direction of the alteration in our study. Moreover, we expected that any observed altered functional connectivity in TD would be associated with disease severity.

\section{MATERIALS AND METHODS}

\section{Participants}

Twenty-three TD patients and $22 \mathrm{HC}$ participated in the study. TD patients were recruited from the Dutch TD patient organization and the outpatient clinics of Altrecht Academic Anxiety Center. HC were recruited from a previously examined cohort (de Wit et al., 2012; de Vries et al., 2014; van Velzen et al., 2014). The Structured Clinical Interview for DSM-IV-TR (SCID-I) on axis I psychiatric disorders was used to screen all participants on in- and exclusion criteria (First et al., 2002). The diagnosis of TD was established by an expert psychiatrist, and a trained research assistant assessed disease severity using the Yale Global Tic Severity Scale (YGTSS) (Leckman et al., 1989). The Yale-Brown Obsessive Compulsive Scale (YBOCS, including both symptom list and severity scale) (Goodman et al., 1989) and the Obsessive-Compulsive Inventory-Revised (OCI-R) (Foa et al., 2002) were administered to assess obsessive-compulsive symptoms and severity in TD patients with comorbidity of obsessive-compulsive disorder (OCD). We used the Beck Depression Inventory (BDI) and Beck Anxiety Inventory (BAI) to assess participants' mood and anxiety symptoms, respectively (Beck et al., 1988, 1996). 
Patients were allowed to have psychiatric comorbidities (except for current or past psychotic disorder, addictions, or mental deficiency), as long as they had a primary diagnosis of TD. Furthermore, participants were excluded if they had a major neurological illness, MRI contra-indications, or insufficient command of the Dutch language. According to the SCID-I, 5 out of the 23 patients with primary diagnosis of TD also met criteria for (OCD). No HC had a current DSM-IV-TR axis-I disorder or a family history of TD. Nine TD patients currently used typical/atypical antipsychotic drugs. The current study was approved by the local ethical review board of the Amsterdam UMC, location VUmc, and all participants provided written informed consent.

\section{Behavioral Analyses}

Demographic, clinical and behavioral measures were analyzed using IBM SPSS Statistics version 22 (IBM, Armonk, NY, United States), using two-sample $t$-tests (2-group comparison between TD patients and HC). Results were considered significant at $p<0.05$, two-tailed. Non-parametric tests were used if data did not meet parametric assumptions.

\section{Image Acquisition}

Imaging data were obtained from a 3-Tesla GE Discovery MR750 scanner (General Electric, Milwaukee, WI, United States) at the Amsterdam UMC, location VUmc equipped with an eightchannel phased-array head coil. Structural images were acquired using a three-dimensional T1-weighted sequence $(256 \times 256$ matrix; voxel size $=1 \mathrm{~mm} \times 0.977 \mathrm{~mm} \times 0.977 \mathrm{~mm}$; 172 sections). Two hundred functional images were acquired using a T2* -weighted single-shot gradient echo-planar imaging sequence $(\mathrm{TR}=1,800 \mathrm{~ms} ; \mathrm{TE}=35 \mathrm{~ms} ; 64 \times 64$ matrix; field of view $=21.1 \mathrm{~cm}$; flip angle $=80^{\circ}$ ) with 39 slices per volume $(3.75 \mathrm{~mm} \times 3.75 \mathrm{~mm}$ in-plane resolution; slice thickness $=3.0 \mathrm{~mm}$; interslice gap $=0.3 \mathrm{~mm}$ ) for whole-brain coverage.

\section{Functional Connectivity Analysis Pre-processing}

The Brain Extraction Tool (BET) from FSL 5.08 ${ }^{1}$ (Smith, 2002) was first applied to remove non-brain tissue from the T1weighted structural images. For the functional data, the first two volumes from the time-series per subject were removed in order to allow for magnetization saturation and enhance data sensitivity. We used Melodic 3.14 (FSL) for an ICA-based singlesession denoising approach (Beckmann et al., 2005) using the following standard processing steps: high-pass filtering (100 s), motion correction with MCFLIRT (Jenkinson et al., 2002), voxelwise demeaning and normalization of the voxel-wise variance, slice timing correction and spatial smoothing with high-pass filtering conducted using a Gaussian kernel of full width at half maximum $(\mathrm{FWHM})=5.0 \mathrm{~mm}$. Average frame-wise displacement (FD) for each subject group was calculated to assess movement. Subjects were excluded if their movements exceeded $3 \mathrm{~mm}$. Pre-processed and filtered functional data were co-registered

${ }^{1}$ https://fsl.fmrib.ox.ac.uk/fsl with structural images by using FLIRT for optimization and registered into Montreal Neurological Institute (MNI) standard space in a two-step procedure, using 7 and 12 degrees of freedom, respectively. FSL FIX $1.061^{2}$, a method that auto-classifies ICA components as veridical or artifactual was applied to detect and remove noise from the time-series (i.e., noise components and motion confounds from the preprocessed dataset) for each subject. Prior to applying FIX, mean relative RMS (root mean squared) displacement (gross motion) from all subjects was examined.

\section{Independent Component Analysis}

We conducted multi-session temporal concatenation ICA on the denoized data sets, with dimensionality set to 20 (numbers of networks extracted from the analysis) to analyze ICAs at the group level. Five masks were created from the group ICAs with threshold $Z=3.0$ : (1) DMN, (2) left FPN, (3) right FPN, (4) $\mathrm{SMN}$, and (5) SN (Figure 1). Dual regression analyses (Filippini et al., 2009) were performed to examine group differences between TD and HC; followed by permutation-based testing (5,000 permutations) using Randomize (Winkler et al., 2014), and Threshold-Free Cluster Enhancement (Smith and Nichols, 2009). A TFCE-corrected $p<0.05$ was considered significant, and $0.05<p<0.09$ was considered as a trend. Age and gender were controlled when added as covariates. Dual regression analyses were also conducted to access potential comorbidity effect [between "pure" (without comorbidity of OCD) and HC] and medication effect (between non-medicated TD and HC).

Within-group multiple regression analyses were conducted to investigate the relationship between the resting state network voxelwise strength and the patients' tic severity (i.e., YGTSS).

\section{Post hoc Analysis on Inter-Network Functional Coupling}

We followed up the main findings from the within-network functional connectivity analysis by investigating if the networks that showed between-group differences differed in their connectivity between networks. FSL's dual regression was used to calculate subject-specific orthogonal time-series and spatial maps for DMN, left FPN and SN, which were defined by ICA (Filippini et al., 2009; Cole et al., 2010; Janes et al., 2012, 2014). We used the $\mathrm{SN}$ as a control network to establish the specificity of functional coupling between the DMN and the FPN. To examine functional coupling between the networks, we extracted the subject-specific time-series for each network per subject and calculated the correlation (Pearson's $r$ ) of these time-series between the DMN and left the FPN, the DMN and the SN, the left FPN and the SN. The values were Fisher-z-transformed for use in further regression and correlational analyses. One-sample $t$-tests were first conducted on Fisher-Z coefficients across all participants to examine the presence and characteristics of functional coupling between each of the two networks (i.e., $t$-value is significantly positive $=$ positive coupling; $t$-value is significantly negative = negative coupling; non-significant $t$-value $=$ no coupling). This was followed up by independent

${ }^{2}$ https://fsl.fmrib.ox.ac.uk/fsl/fslwiki/FIX/ 


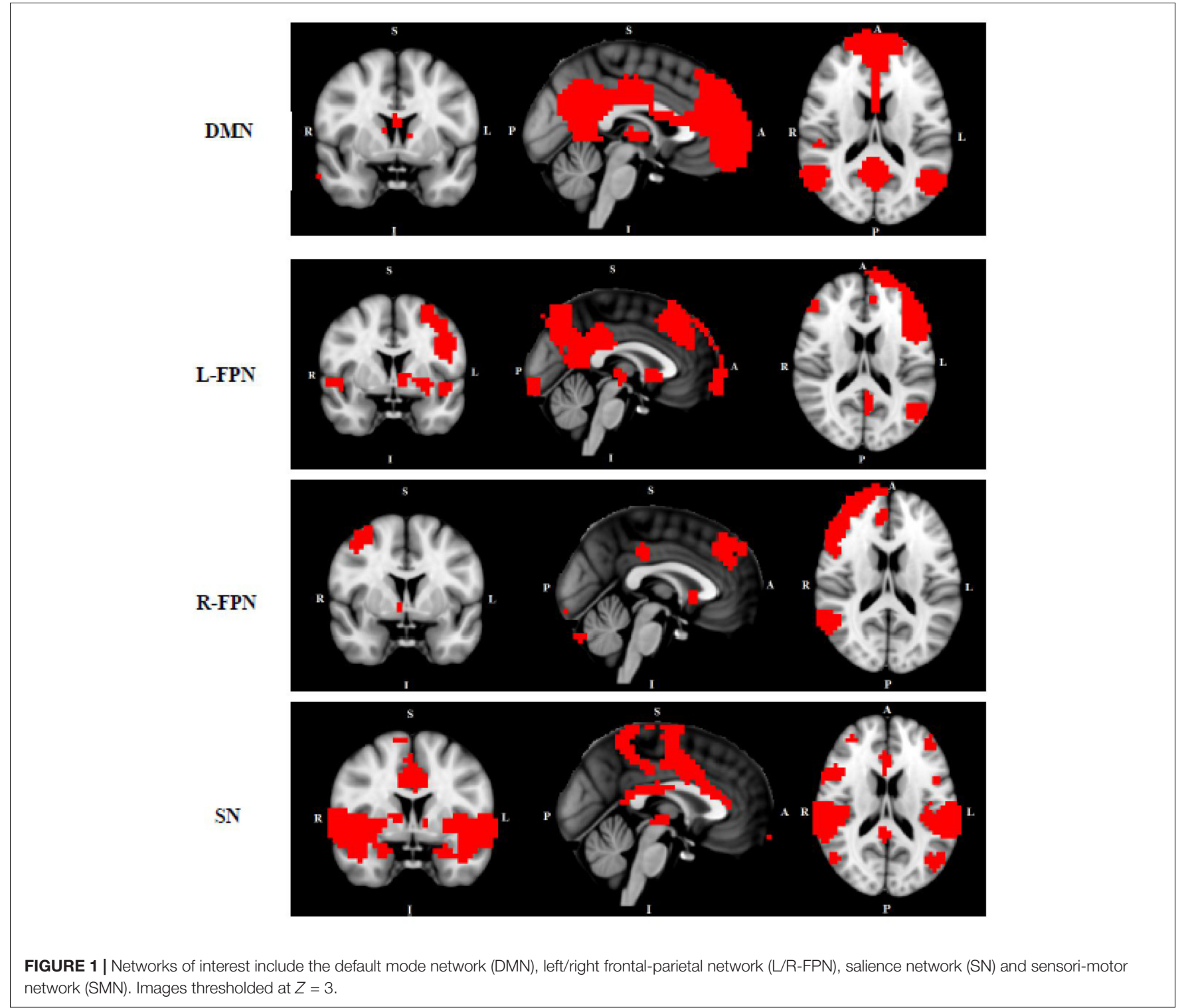

samples $t$-tests for between-group comparisons on any identified functional coupling between networks (Putcha et al., 2015).

\section{RESULTS}

\section{Demographic and Clinical Characteristics}

There was no difference of $\mathrm{FD}$ between $\mathrm{TD}$ (mean = $0.067 \pm 0.050 \mathrm{~mm})$ and HC $($ mean $=0.066 \pm 0.021 \mathrm{~mm})$. No subjects showed excessive movement $(>3 \mathrm{~mm}$ ) and there were no differences in movements between two subject groups, therefore all were included. Demographic and clinical characteristics of participants included in the between-group comparison (TD patients and HC) are shown in Table 1. No group differences were found on gender and educational level while on average the TD patients (mean age $34.6 \pm 12.3$ years) were younger than $\mathrm{HC}$ (mean age $43.3 \pm 14.2$ years). The patient group scored higher on TD severity, BDI and BAI than HC.

\section{Functional Connectivity of Resting-State Networks}

The dual regression approach allows identifying which areas within a given network differ between TD patients and $\mathrm{HC}$ in terms of the strength of their contribution to the independent component, reflecting their level of connectivity to the rest of that network. We therefore refer to our findings as indices of functional connectivity in the below. This approach showed a significantly higher involvement of the left dorsal medial prefrontal cortex in the neural functional connectivity of the DMN in TD patients as compared to HC $(p<0.05)$ (Figure 2 and Table 2). A significantly lower degree of involvement in the functional connectivity of the left FPN was also found in TD patients compared to $\mathrm{HC}$ at the left inferior parietal cortex 
TABLE 1 | Demographic and clinical measures of the study samples: including patients with Tourette's disorder [with comorbidity of obsessive compulsive disorder (OCD)] and healthy controls.

\begin{tabular}{|c|c|c|c|c|c|}
\hline \multirow[b]{2}{*}{ Demographic measures } & \multicolumn{2}{|c|}{$\begin{array}{c}\text { TD } \\
(N=23)\end{array}$} & \multicolumn{2}{|c|}{$\begin{array}{c}\mathrm{HC} \\
(N=22)\end{array}$} & \multirow[b]{2}{*}{$p$} \\
\hline & $N$ & $\%$ & $N$ & $\%$ & \\
\hline \multirow[t]{2}{*}{ Gender (men) } & 15 & 65 & 14 & 64 & 0.59 \\
\hline & Mean & $S D$ & Mean & $S D$ & $p$ \\
\hline Age (years) & 34.6 & 12.3 & 43.3 & 14.2 & 0.035 \\
\hline Education levela & 8.1 & 1.7 & 8.2 & 2.0 & $0.74^{b}$ \\
\hline \multicolumn{6}{|l|}{ Clinical measures } \\
\hline YGTSS (range 0-100) & 46.3 & 16.7 & $\mathrm{~N} / \mathrm{A}$ & $\mathrm{N} / \mathrm{A}$ & \\
\hline Y-BOCS severity (range 0-40) & $16.8(N=5)$ & 6.3 & $\mathrm{~N} / \mathrm{A}$ & $\mathrm{N} / \mathrm{A}$ & \\
\hline $\mathrm{BDI}$ & 6.7 & 6.6 & 2.1 & 3.0 & $0.002^{b}$ \\
\hline $\mathrm{BAI}$ & 7.8 & 7.3 & 2.1 & 2.8 & $<0.001^{\mathrm{b}}$ \\
\hline
\end{tabular}

aducational level was recorded in nine levels ranging from 1 (no finished education) to 9 (university training). ${ }^{b}$ Mann-Whitney $U$ test. YGTSS, Yale Globe Tic Severity Scale; Y-BOCS, Yale-Brown Obsessive Compulsive Scale; BDI, Beck Depression Inventory; BAl, Beck Anxiety Inventory; TD, Tourette's disorder; OCD, obsessivecompulsive disorder; HCs, healthy controls.

(IPC) region $(p<0.05)$ to the rest of the network (Figure 3 and Table 2). The above results remained significant when only "pure" TD patients (without comorbidity of OCD; $N=18$ ) were compared to HC. A trend-higher functional connectivity in the DMN (from the same dmPFC region to rest of the DMN) was found when only the unmedicated TD patients $(N=13)$ were compared to $\mathrm{HC}(p=0.09)$, while a significantly lower functional connectivity in the left FPN (from the same left IPC region to rest of the left FPN) remained. No differences were observed between TD and HC in any other targeted networks.

\section{Post hoc Analysis on Inter-Network Functional Coupling}

A positive coupling between the DMN and the left FPN was revealed with the overall mean Fisher- $Z$ coefficients across TD patients and $\mathrm{HC}$ showing statistical significance (Mean $\mathrm{r}$ square $=0.29, S E=0.03),[t(44)=8.78, p<0.001]$; while there was a significantly negative coupling between DMN and $\mathrm{SN}[$ Mean $=-0.23, S E=0.04, t(44)=-6.34, p<0.001]$. No coupling was found between the left FPN and the SN. When compared with controls, TD patients showed a stronger positive coupling between the DMN and the left FPN $(t=-2.58, p=0.01)$ (Figure 4).

\section{Correlations Between Functional Connectivity and Clinical Characteristics}

In the within-group regression analyses (in TD patients only), functional connectivity in the DMN correlated negatively with tic severity $(p<0.05)$ (Figure 5).

\section{DISCUSSION}

The current study investigated functional connectivity in TD in four resting-state networks, i.e., the DMN, FPN, SMN, and $\mathrm{SN}$. Within the DMN, TD patients compared to HC showed a higher connectivity between the left dmPFC and the rest of the network. Within the left FPN, TD patients compared to

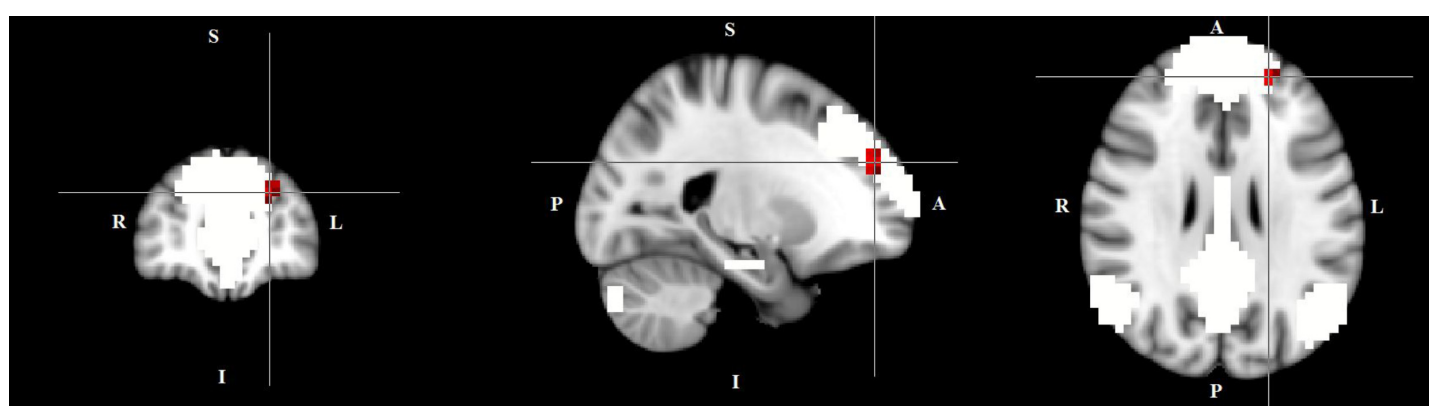

FIGURE 2 | Higher functional connectivity in TD patients compared to HC in the default mode network. The image displays a higher involvement of the left dorsal medial prefrontal cortex region in the neural functional connectivity to the rest of the default mode network in TD patients $(X=-22, Y=49, Z=28, p=0.0056)$. For illustration purposes, $p$ thresholded at 0.05 .

TABLE 2 | Regions of significant difference between patients with Tourette's disorder (TD) and healthy controls.

Two-group comparisons

Coordinates (MNI)

\begin{tabular}{|c|c|c|c|c|c|c|}
\hline Resting-state networks & Region & $x$ & $y$ & $z$ & Cluster size & $p$ \\
\hline Default-mode network & Left dorsal medial prefrontal cortex & -22 & 49 & 28 & 38 & 0.0056 \\
\hline Left frontal-parietal network & Left inferior parietal cortex & -46 & -69 & 29 & 20 & 0.0186 \\
\hline
\end{tabular}

The table includes all regions that met the Threshold-Free Cluster Enhancement significance level, corrected for $p<0.05$. MNI, Montreal Neurological Institute. 


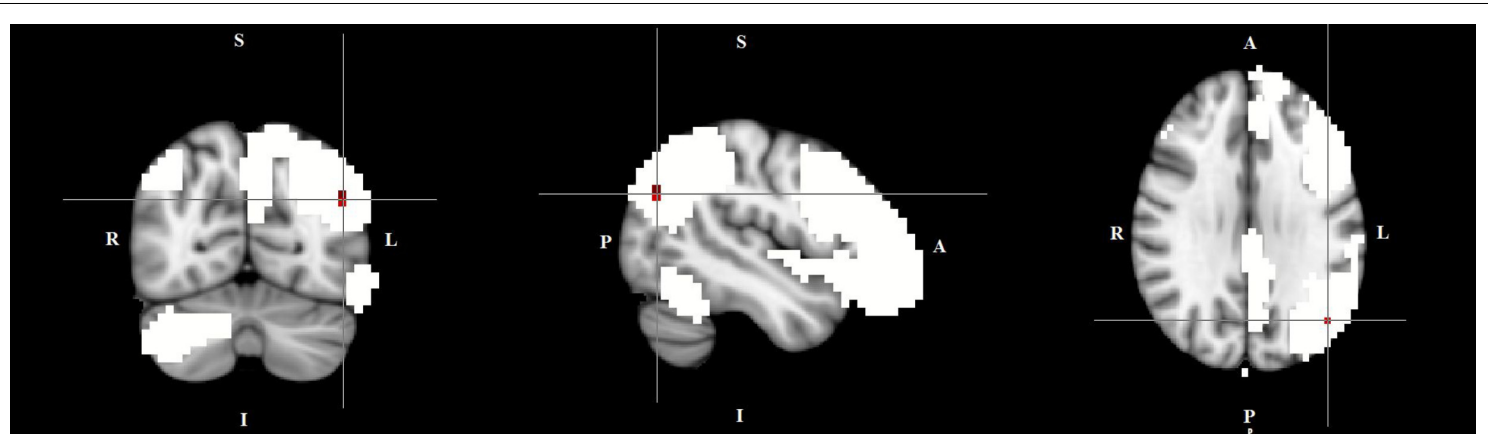

FIGURE 3 | Lower functional connectivity in TD patients compared to $\mathrm{HC}$ in the left frontal-parietal network. The image displays a lower degree of involvement of the left inferior parietal cortex region in the neural functional connectivity to the rest of the left frontal-parietal network in TD patients $(X=-46, Y=-69, Z=29$, $p=0.0186)$. For illustration purposes, $p$ thresholded at 0.05 .

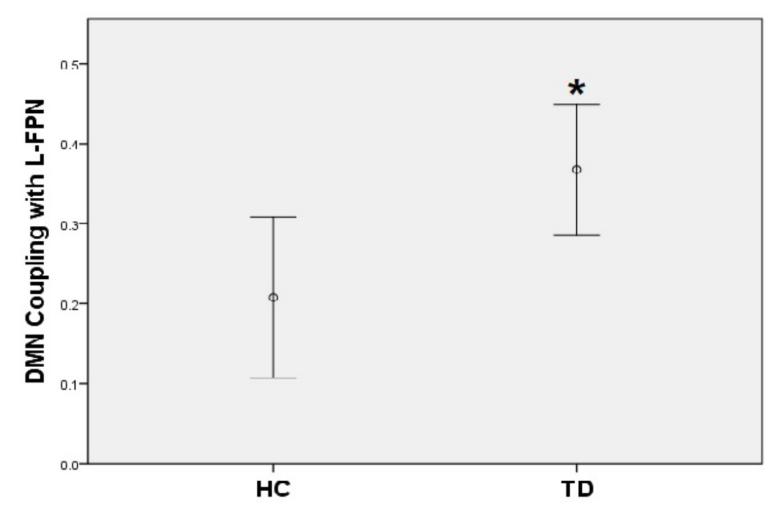

FIGURE 4 | TD patients showed significantly higher positive coupling between default mode network and left frontal-parietal network compared to $\mathrm{HC}$ $(* p<0.05)$.

HC showed significantly lower connectivity between the left IPC region and the rest of the network. Also, TD patients compared with $\mathrm{HC}$ showed stronger coupling between the DMN and the left FPN. In TD, functional connectivity in the left dmPFC to rest of the DMN correlated negatively with tic severity.
Although the DMN finding appears to be at odds with Neuner et al. (2014), who reported 'relatively preserved' connectivity within the DMN in TD patients during free 'ticcing' during scanning, the two studies are not comparable due to different conditions. However, since our study subjects were instructed to lie still during scanning, we cannot rule out the possibility that the study findings may reflect an effect of tic suppression rather than a true resting-state in our TD patients. The dmPFC is suggested to be one of the highest associative centers in the frontal lobe participating in cognitive processes and autobiographical retrieval. In TD, thinner cortex of the dmPFC has been found to relate to more severe tic symptoms (Sowell et al., 2008), and lower white matter volume has also been demonstrated in this area (Greene et al., 2017). The dmPFC is a key node of the DMN, which is particularly relevant for processing highly demanding and complex cognitive tasks (Eickhoff et al., 2016). Although the DMN has been implicated in mind-wandering (Mason et al., 2007) and monitoring the world around us (Gusnard et al., 2001), it has also been linked to integrating cognitive processing (Greicius et al., 2003). Due to long-term struggling and coping with inappropriate acts, adult TD patients can achieve a variety of cognitive tasks by enhancing cognitive control (Mueller et al., 2006; Jackson et al., 2007). Therefore, although speculative at this stage, the increased dmPFC functional connectivity in the

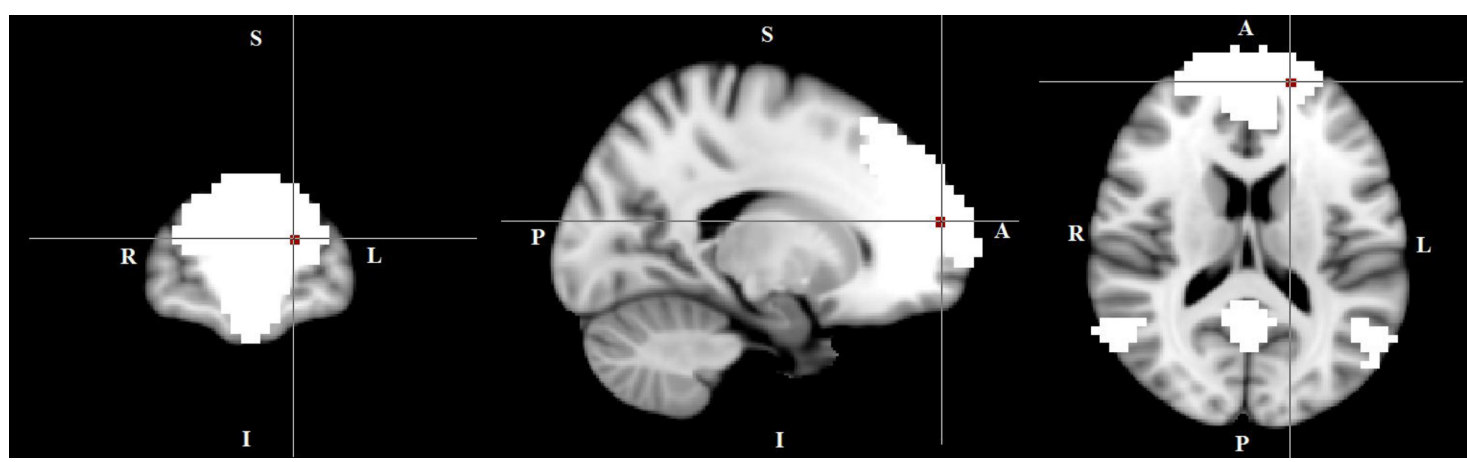

FIGURE 5 | A negative correlation between functional connectivity in the default mode network and tic severity scores in TD patients $(X=-17, Y=59, Z=16$, $p=0.034$ ). For illustration purposes, $p$ was thresholded at 0.05 . 
DMN might be associated with enhanced cognitive control as a compensatory cognitive mechanism developed by our adult TD patients as a result of long-term tic suppression.

Tourette's disorder patients showed decreased functional connectivity in the left IPC within the FPN network compared to HC. The IPC is known for its role in attention processes, motor control and response inhibition (Rizzolatti et al., 1997; Rushworth et al., 2001; Cieslik et al., 2015; Fan et al., 2017). In TD, lower cortical thickness of the IPC was found to associate with more severe tic symptoms (Fan et al., 2017). Functionally, tic genesis corresponds to abnormal activation in the parietal cortex (the IPC and superior parietal cortex) (Stern et al., 2000; Bohlhalter et al., 2006), and higher IPC activity was found during successful inhibition in TD patients (Fan et al., 2017). The current study, although using a different methodological approach compared with other studies, shows results that are in line with two previous studies reporting abnormal functional connectivity within the FPN in adolescent and adult TD patients (Church et al., 2009; Worbe et al., 2012). The FPN is known for its role in attention processing (Biswal et al., 1995) and subserves attentional gating, shifting focus of interest (Dehaene and Naccache, 2001), and maintaining information content that is necessary for performance on online rapid adaptive control tasks (Dosenbach et al., 2008).

Anatomically, the FPN is closely connected with the DMN (Vincent et al., 2008) and the FPN co-activates with the DMN for production of internal thoughts (Smallwood et al., 2012). It has been proposed that the FPN serves as an attention control network that cooperates with the DMN to aid in either internal thought coordination or sustaining the internal thought stream and preventing it from disruption of external stimuli (Smallwood et al., 2012). In our follow-up internetwork connectivity analyses, findings were consistent with the suggestion of Smallwood et al. (2012), in which positive coupling between the DMN and the FPN across TD and HC was revealed. Additionally, we used the $\mathrm{SN}$ as a control network and investigated its functional coupling with both the DMN and the left FPN to further study whether this observed positive inter-network coupling was specific or a general feature of all four networks. The SN was negatively coupled with the DMN while no coupling was found between the $\mathrm{SN}$ and the FPN. Between-group comparisons showed that TD patients have significantly stronger coupling between the DMN and the FPN than HC.

Dysfunction of the FPN contributes to TD pathophysiology (Church et al., 2009; Worbe et al., 2012), possibly reflecting an inefficient ability of the FPN to prevent the disruption of internal thought streams that are generated by the DMN by external stimuli. Although speculative, one possible interpretation of our main findings might be that adult TD patients are able to boost their DMN functional connectivity. Such a suggestion seems to agree with our observed negative correlation between functional connectivity and tic severity in the DMN, such that a higher DMN activity is associated with a better tic suppression. It remains to be shown, nevertheless, whether the DMN plays an active role in the control of ticcing behavior.
No group differences between TD and $\mathrm{HC}$ were observed in the SMN and the SN. This is partly inconsistent with previous literature showing overall disorganizations in functional connectivity between cortical, basal ganglia and limbic regions in TD (Church et al., 2009; Werner et al., 2010; Worbe et al., 2012). We suspect that the absence of alterations in the SMN and the SN might reflect differences in methodological approaches used. Another explanation for the negative findings might be a lack of power of our study to identify potential differences between TD and controls because of the modest sample size in this study.

The current study has several additional limitations including: (1) difference in age between TD and HC groups; (2) the use of medication in some of our TD patients; (3) an a priori selection of networks of interest; (4); although each regression analysis was corrected for multiple comparisons using TFCE, we did not correct for the number of analyses in order to avoid false negative findings. Therefore, replication is needed in larger, independent and well-matched samples with sophisticated experimental designs to control for a possible tic-suppression effect in the future.

\section{CONCLUSION}

In conclusion, compared with $\mathrm{HCs}$, TD patients showed increased connectivity in the DMN and decreased connectivity in the left FPN, respectively, combined with a stronger positive functional coupling between these two resting-state networks. In addition, higher functional connectivity within the DMN was associated with a lower tic severity. Taken together, our results suggest that TD patients show enhanced functional coupling between the DMN and the FPN in order to compensate for altered functional connectivity within these two networks. This might be a compensatory mechanism to overcome motor tics.

\section{AUTHOR CONTRIBUTIONS}

SF contributed to data collection, research analyses, and manuscript written and edited. $\mathrm{OH}$ and DC contributed to study design and manuscript edited. SW contributed to manuscript edited. CV contributed to part of research analyses and manuscript edited. DV contributed to study design, part of research analyses, and manuscript edited. YW contributed to study design, part of research analyses, and manuscript edited.

\section{FUNDING}

This study was supported by FP7-People-2012-ITN grant (316978, project: TS-Eurotrain).

\section{ACKNOWLEDGMENTS}

The authors thank Karlijn Schols, M.Sc., Ires Ghielen M.Sc., Tim van Balkom, M.Sc., Sonja Rutten, M.D., and Mardien Oudega, M.D., Ph.D. for their contributions to data collection. 


\section{REFERENCES}

Alexander, G. E., DeLong, M. R., and Strick, P. L. (1986). Parallel organization of functionally segregated circuits linking basal ganglia and cortex. Annu. Rev. Neurosci. 9, 357-381. doi: 10.1146/annurev.ne.09.030186.002041

Beck, A. T., Epstein, N., Brown, G., and Steer, R. A. (1988). An inventory for measuring clinical anxiety: psychometric properties. J. Consult. Clin. Psychol. 56, 893-897. doi: 10.1037/0022-006X.56.6.893

Beck, A. T., Steer, R. A., Ball, R., and Ranieri, W. (1996). Comparison of beck depression inventories -IA and -II in psychiatric outpatients. J. Pers. Assess 67, 588-597. doi: 10.1207/s15327752jpa6703_13

Beckmann, C. F., DeLuca, M., Devlin, J. T., and Smith, S. M. (2005). Investigations into resting-state connectivity using independent component analysis. Philos. Trans. R. Soc. Lond. B Biol. Sci 360, 1001-1013. doi: 10.1098/rstb.2005.1634

Biswal, B., Yetkin, F. Z., Haughton, V. M., and Hyde, J. S. (1995). Functional connectivity in the motor cortex of resting human brain using echo-planar MRI Magn. Reson. Med. 34, 537-541. doi: 10.1002/mrm.1910340409

Biswal, B. B., Van Kylen, J., and Hyde, J. S. (1997). Simultaneous assessment of flow and BOLD signals in resting-state functional connectivity maps. NMR Biomed 10, 165-170. doi: 10.1002/(SICI)1099-1492(199706/08)10:4/5<165:: AID-NBM454>3.0.CO;2-7

Bohlhalter, S., Goldfine, A., Matteson, S., Garraux, G., Hanakawa, T., Kansaku, K., et al. (2006). Neural correlates of tic generation in Tourette syndrome: an event-related functional MRI study. Brain 129, 2029-2037. doi: 10.1093/brain/ awl050

Buckner, R. L., and Vincent, J. L. (2007). Unrest at rest: default activity and spontaneous network correlations. Neuroimage 37, 1091-6; discussion 10971099. doi: 10.1016/j.neuroimage.2007.01.010

Church, J. A., Fair, D. A., Dosenbach, N. U., Cohen, A. L., Miezin, F. M., Petersen, S. E., et al. (2009). Control networks in paediatric Tourette syndrome show immature and anomalous patterns of functional connectivity. Brain 132, 225-238. doi: 10.1093/brain/awn223

Cieslik, E. C., Mueller, V. I., Eickhoff, C. R., Langner, R., and Eickhoff, S. B. (2015). Three key regions for supervisory attentional control: evidence from neuroimaging meta-analyses. Neurosci. Biobehav. Rev. 48, 22-34. doi: 10.1016/ j.neubiorev.2014.11.003

Cole, D. M., Beckmann, C. F., Long, C. J., Matthews, P. M., Durcan, M. J., and Beaver, J. D. (2010). Nicotine replacement in abstinent smokers improves cognitive withdrawal symptoms with modulation of resting brain network dynamics. Neuroimage 52, 590-599. doi: 10.1016/j.neuroimage.2010.04.251

Damoiseaux, J. S., Rombouts, S. A., Barkhof, F., Scheltens, P., Stam, C. J., Smith, S. M., et al. (2006). Consistent resting-state networks across healthy subjects. Proc. Natl. Acad. Sci. U.S.A 103, 13848-13853. doi: 10.1073/pnas.0601417103

De La Fuente, A., Xia, S., Branch, C., and Li, X. (2013). A review of attentiondeficit/hyperactivity disorder from the perspective of brain networks. Front. Hum. Neurosci. 7:192. doi: 10.3389/fnhum.2013.00192

de Vries, F. E., de Wit, S. J., Cath, D. C., van der Werf, Y. D., van der Borden, V., van Rossum, T. B., et al. (2014). Compensatory frontoparietal activity during working memory: an endophenotype of obsessive-compulsive disorder. Biol. Psychiatry 76, 878-887. doi: 10.1016/j.biopsych.2013.11.021

de Wit, S. J., de Vries, F. E., van der Werf, Y. D., Cath, D. C., Heslenfeld, D. J., Veltman, E. M., et al. (2012). Presupplementary motor area hyperactivity during response inhibition: a candidate endophenotype of obsessive-compulsive disorder. Am. J. Psychiatry 169, 1100-1108. doi: 10.1176/appi.ajp.2012. 12010073

Dehaene, S., and Naccache, L. (2001). Towards a cognitive neuroscience of consciousness: basic evidence and a workspace framework. Cognition 79, 1-37. doi: 10.1016/S0010-0277(00)00123-2

Dosenbach, N. U., Fair, D. A., Cohen, A. L., Schlaggar, B. L., and Petersen, S. E. (2008). A dual-networks architecture of top-down control. Trends Cogn. Sci. 12, 99-105. doi: 10.1016/j.tics.2008.01.001

Eickhoff, S. B., Laird, A. R., Fox, P. T., Bzdok, D., and Hensel, L. (2016). Functional Segregation of the Human Dorsomedial Prefrontal Cortex. Cereb. Cortex 26, 304-321. doi: 10.1093/cercor/bhu250

Esposito, F., Tessitore, A., Giordano, A., De Micco, R., Paccone, A., Conforti, R., et al. (2013). Rhythm-specific modulation of the sensorimotor network in drugnaive patients with Parkinson's disease by levodopa. Brain 136, 710-725. doi: 10.1093/brain/awt007
Fan, S., Cath, D. C., van der Werf, Y. D., de Wit, S., Veltman, D. J., and van den Heuvel, O. A. (2017). Trans-diagnostic comparison of response inhibition in Tourette's disorder and obsessive-compulsive disorder. World J. Biol. Psychiatry doi: 10.1080/15622975.2017.1347711 [Epub ahead of print].

Filippini, N., MacIntosh, B. J., Hough, M. G., Goodwin, G. M., Frisoni, G. B., Smith, S. M., et al. (2009). Distinct patterns of brain activity in young carriers of the APOE-epsilon4 allele. Proc. Natl. Acad. Sci. U.S.A 106, 7209-7214. doi: 10.1073/pnas.0811879106

First, M. B., Gibbon, M. L., and Williams, J. B. W. (2002). Structured Clinical Interview for DSM-IV-TR Axis I Disorders, Research Version, Patient Edition (SCID-I/P). New York, NY: New York State Psychiatric Institute.

Foa, E. B., Huppert, J. D., Leiberg, S., Langner, R., Kichic, R., Hajcak, G., et al. (2002). The Obsessive-Compulsive Inventory: development and validation of a short version. Psychol. Assess. 14, 485-496. doi: 10.1037/1040-3590.14.4.485

Freeman, M. L. (2013). American Pancreatic Association presidential address: widening the reach of the APA. Pancreas 42, 1201-1202. doi: 10.1097/MPA. 0b013e3182a6e093

Ganos, C., Roessner, V., and Munchau, A. (2013). The functional anatomy of Gilles de la Tourette syndrome. Neurosci. Biobehav. Rev. 37, 1050-1062. doi: 10.1016/j.neubiorev.2012.11.004

Goodman, W. K., Price, L. H., Rasmussen, S. A., Mazure, C., Delgado, P., Heninger, G. R., et al. (1989). The yale-brown obsessive compulsive scale. II. Validity. Arch. Gen. Psychiatry 46, 1012-1016. doi: 10.1001/archpsyc.1989.01810110054008

Greene, D. J., Williams, A. C. I. I. I., Koller, J. M., Schlaggar, B. L., and Black, K. J. (2017). Brain structure in pediatric Tourette syndrome. Mol. Psychiatry 22, 972-980. doi: 10.1038/mp.2016.194

Greicius, M. D., Krasnow, B., Reiss, A. L., and Menon, V. (2003). Functional connectivity in the resting brain: a network analysis of the default mode hypothesis. Proc. Natl. Acad. Sci. U.S.A 100, 253-258. doi: 10.1073/pnas. 0135058100

Gusnard, D. A., Raichle, M. E., and Raichle, M. E. (2001). Searching for a baseline: functional imaging and the resting human brain. Nat. Rev. Neurosci. 2, 685-694. doi: $10.1038 / 35094500$

Ham, T., Leff, A., de Boissezon, X., Joffe, A., and Sharp, D. J. (2013). Cognitive control and the salience network: an investigation of error processing and effective connectivity. J. Neurosci. 33, 7091-7098. doi: 10.1523/JNEUROSCI. 4692-12.2013

Jackson, G. M., Mueller, S. C., Hambleton, K., and Hollis, C. P. (2007). Enhanced cognitive control in Tourette Syndrome during task uncertainty. Exp. Brain Res. 182, 357-364. doi: 10.1007/s00221-007-0999-8

Janes, A. C., Farmer, S., Frederick, B., Nickerson, L. D., and Lukas, S. E. (2014). An increase in tobacco craving is associated with enhanced medial prefrontal cortex network coupling. PLoS One 9:e88228. doi: 10.1371/journal.pone.0088228

Janes, A. C., Nickerson, L. D., Frederick, B. B., and Kaufman, M. J. (2012). Prefrontal and limbic resting state brain network functional connectivity differs between nicotine-dependent smokers and non-smoking controls. Drug Alcohol Depend 125, 252-259. doi: 10.1016/j.drugalcdep.2012.02.020

Jenkinson, M., Bannister, P., Brady, M., and Smith, S. (2002). Improved optimization for the robust and accurate linear registration and motion correction of brain images. Neuroimage 17, 825-841. doi: 10.1006/nimg.2002. 1132

Ji, G. J., Liao, W., Yu, Y., Miao, H. H., Feng, Y. X., Wang, K., et al. (2016) Globus pallidus interna in tourette syndrome: decreased local activity and disrupted functional connectivity. Front. Neuroanat. 10:93. doi: 10.3389/fnana. 2016.00093

Leckman, J. F. (2002). Tourette's syndrome. Lancet 360, 1577-1586. doi: 10.1016 S0140-6736(02)11526-1

Leckman, J. F., Riddle, M. A., Hardin, M. T., Ort, S. I., Swartz, K. L., Stevenson, J., et al. (1989). The yale global tic severity scale: initial testing of a clinicianrated scale of tic severity. J. Am. Acad. Child Adolesc. Psychiatry 28, 566-573. doi: 10.1097/00004583-198907000-00015

Liao, W., Yu, Y., Miao, H. H., Feng, Y. X., Ji, G. J., and Feng, J. H. (2017). Interhemispheric intrinsic connectivity as a neuromarker for the diagnosis of boys with tourette syndrome. Mol. Neurobiol. 54, 2781-2789. doi: 10.1007/s12035016-9863-9

Mason, M. F., Norton, M. I., Van Horn, J. D., Wegner, D. M., Grafton, S. T., and Macrae, C. N. (2007). Wandering minds: the default network and stimulusindependent thought. Science 315, 393-395. doi: 10.1126/science.1131295 
Milad, M. R., and Rauch, S. L. (2012). Obsessive-compulsive disorder: beyond segregated cortico-striatal pathways. Trends Cogn. Sci. 16, 43-51. doi: 10.1016/ j.tics.2011.11.003

Mueller, S. C., Jackson, G. M., Dhalla, R., Datsopoulos, S., and Hollis, C. P. (2006). Enhanced cognitive control in young people with Tourette's syndrome. Curr. Biol. 16, 570-573. doi: 10.1016/j.cub.2006.01.064

Neuner, I., Werner, C. J., Arrubla, J., Stocker, T., Ehlen, C., Wegener, H. P., et al. (2014). Imaging the where and when of tic generation and resting state networks in adult Tourette patients. Front. Hum. Neurosci. 8:362. doi: 10.3389/fnhum. 2014.00362

Putcha, D., Ross, R. S., Cronin-Golomb, A., Janes, A. C., and Stern, C. E. (2015). Altered intrinsic functional coupling between core neurocognitive networks in Parkinson's disease. Neuroimage Clin. 7, 449-455. doi: 10.1016/j.nicl.2015. 01.012

Rizzolatti, G., Fogassi, L., and Gallese, V. (1997). Parietal cortex: from sight to action. Curr. Opin. Neurobiol. 7, 562-567. doi: 10.1016/S0959-4388(97)80037-2

Rushworth, M. F., Paus, T., and Sipila, P. K. (2001). Attention systems and the organization of the human parietal cortex. J. Neurosci. 21, 5262-5271. doi: 10.1523/JNEUROSCI.21-14-05262.2001

Seeley, W. W., Menon, V., Schatzberg, A. F., Keller, J., Glover, G. H., Kenna, H., et al. (2007). Dissociable intrinsic connectivity networks for salience processing and executive control. J. Neurosci. 27, 2349-2356. doi: 10.1523/JNEUROSCI. 5587-06.2007

Smallwood, J., Brown, K., Baird, B., and Schooler, J. W. (2012). Cooperation between the default mode network and the frontal-parietal network in the production of an internal train of thought. Brain Res. 1428, 60-70. doi: 10.1016/ j.brainres.2011.03.072

Smith, S. M. (2002). Fast robust automated brain extraction. Hum. Brain Mapp. 17, 143-155. doi: 10.1002/hbm.10062

Smith, S. M., and Nichols, T. E. (2009). Threshold-free cluster enhancement: addressing problems of smoothing, threshold dependence and localisation in cluster inference. Neuroimage 44, 83-98. doi: 10.1016/j.neuroimage.2008. 03.061

Sowell, E. R., Kan, E., Yoshii, J., Thompson, P. M., Bansal, R., Xu, D., et al. (2008). Thinning of sensorimotor cortices in children with Tourette syndrome. Nat. Neurosci. 11, 637-639. doi: 10.1038/nn.2121

Stern, E., Silbersweig, D. A., Chee, K. Y., Holmes, A., Robertson, M. M., Trimble, M., et al. (2000). A functional neuroanatomy of tics in Tourette syndrome. Arch. Gen. Psychiatry 57, 741-748. doi: 10.1001/archpsyc.57.8.741

Stern, E. R., Fitzgerald, K. D., Welsh, R. C., Abelson, J. L., and Taylor, S. F. (2012). Resting-state functional connectivity between fronto-parietal and default mode networks in obsessive-compulsive disorder. PLoS One 7:e36356. doi: 10.1371/ journal.pone.0036356

Tessitore, A., Esposito, F., Vitale, C., Santangelo, G., Amboni, M., Russo, A., et al. (2012). Default-mode network connectivity in cognitively unimpaired patients with Parkinson disease. Neurology 79, 2226-2232. doi: 10.1212/WNL. 0b013e31827689d6 van den Heuvel, M. P., and Hulshoff Pol, H. E. (2010). Exploring the brain network: a review on resting-state fMRI functional connectivity. Eur. Neuropsychopharmacol. 20, 519-534. doi: 10.1016/j.euroneuro.2010.03.008

van Velzen, L. S., Vriend, C., de Wit, S. J., and van den Heuvel, O. A. (2014). Response inhibition and interference control in obsessive-compulsive spectrum disorders. Front. Hum. Neurosci. 8:419. doi: 10.3389/fnhum.2014. 00419

Vincent, J. L., Kahn, I., Snyder, A. Z., Raichle, M. E., and Buckner, R. L. (2008). Evidence for a frontoparietal control system revealed by intrinsic functional connectivity. J. Neurophysiol. 100, 3328-3342. doi: 10.1152/jn.90355.2008

Wen, H., Liu, Y., Rekik, I., Wang, S., Chen, Z., Zhang, J., et al. (2018). Combining disrupted and discriminative topological properties of functional connectivity networks as neuroimaging biomarkers for accurate diagnosis of early tourette syndrome children. Mol. Neurobiol. 55, 3251-3269. doi: 10.1007/s12035-0170519-1

Werner, C. J., Dogan, I., Sass, C., Mirzazade, S., Schiefer, J., Shah, N. J., et al. (2014). Altered resting-state connectivity in Huntington's disease. Hum. Brain Mapp. 35, 2582-2593. doi: 10.1002/hbm.22351

Werner, C. J., Stocker, T., Kellermann, T., Wegener, H. P., Schneider, F., Shah, N. J., et al. (2010). Altered amygdala functional connectivity in adult Tourette's syndrome. Eur. Arch. Psychiatry Clin. Neurosci. 260(Suppl. 2), S95-S99. doi: 10.1007/s00406-010-0161-7

Winkler, A. M., Ridgway, G. R., Webster, M. A., Smith, S. M., and Nichols, T. E. (2014). Permutation inference for the general linear model. Neuroimage 92, 381-397. doi: 10.1016/j.neuroimage.2014.01.060

Wolf, R. C., Sambataro, F., Vasic, N., Wolf, N. D., Thomann, P. A., Saft, C., et al. (2012). Default-mode network changes in preclinical Huntington's disease. Exp. Neurol 237, 191-198. doi: 10.1016/j.expneurol.2012.06.014

Worbe, Y., Lehericy, S., and Hartmann, A. (2015). Neuroimaging of tic genesis: present status and future perspectives. Mov. Disord. 30, 1179-1183. doi: 10. $1002 /$ mds. 26333

Worbe, Y., Malherbe, C., Hartmann, A., Pelegrini-Issac, M., Messe, A., Vidailhet, M., et al. (2012). Functional immaturity of cortico-basal ganglia networks in Gilles de la Tourette syndrome. Brain 135, 1937-1946. doi: 10.1093/ brain/aws056

Conflict of Interest Statement: The authors declare that the research was conducted in the absence of any commercial or financial relationships that could be construed as a potential conflict of interest.

Copyright (C) 2018 Fan, van den Heuvel, Cath, de Wit, Vriend, Veltman and van der Werf. This is an open-access article distributed under the terms of the Creative Commons Attribution License (CC BY). The use, distribution or reproduction in other forums is permitted, provided the original author(s) and the copyright owner(s) are credited and that the original publication in this journal is cited, in accordance with accepted academic practice. No use, distribution or reproduction is permitted which does not comply with these terms. 